\title{
SOLVABILITY FOR A CLASS OF NONLINEAR \\ CAPUTO-HADAMARD FRACTIONAL DIFFERENTIAL EQUATIONS WITH p-LAPLACIAN OPERATOR IN BANACH SPACES
}

\author{
Choukri Derbazi
} \section{$\mathrm{ND}$}

(C) by University of Niš, Serbia | Creative Commons Licence: CC BY-NC-

Abstract. This paper is devoted to the existence of solutions for certain classes of nonlinear differential equations involving the Caputo-Hadamard fractional-order with pLaplacian operator in Banach spaces. The arguments are based on Mönch's fixed point theorem combined with the technique of measures of noncompactness. An example is also presented to illustrate the effectiveness of the main results.

Keywords: Banach spaces; differential equations; Caputo-Hadamard fractional-order; Laplacian operator; Mönch's fixed point theorem.

\section{Introduction}

Fractional calculus is a branch of mathematical analysis that deals with the derivatives and integrals of arbitrary (non-integer) order. In fact fractional calculus has developed into an important field of research during the last few decades in view of its widespread applications in a variety of disciplines such as physics, chemistry, biology, biophysics, blood flow phenomena, control theory, wave propagation, signal and image processing, viscoelasticity, financial mathematics, economics, etc. (see $[28,32,33,37,38,42,44])$.

Fixed point theory is an important tool in Nonlinear Analysis, in particular, in obtaining existence results for a variety of mathematical problems. Although there are many methods (such as Banach contraction principle, Schauder's fixed point theorem, and Krasnoselskii's fixed point theorem, etc.) to analyze, under suitable conditions, the existence and uniqueness of solution of various problems with initial conditions, boundary conditions, integral boundary conditions, nonlinear boundary conditions, and periodic boundary conditions for fractional differential equations,

Received August 19, 2019; accepted February 26, 2020

2010 Mathematics Subject Classification. Primary 34A08; Secondary 26A33, 34B10 
for more details see for instance $[5,13,18,19,20,21,26,29,43]$. We focus here on the so-called measures of noncompactness, see for instance [1, 2, 4, 12, 14, 39]. A key result in the cited works to prove the existence of solutions is the celebrated Mönch fixed point theorem, based on such measures. We also refer the readers to the recent book [11], where several applications of the measure of noncompactness can be found.

A p-Laplacian differential equation was first introduced by Leibenson [31] when he studied the turbulent flow in a porous medium. Since then, fractional differential equations and the differential equation with a p-Laplacian operator are widely applied in different fields of physics and natural phenomena, for example, non-Newtonian mechanics, fluid mechanics, viscoelasticity mechanics, combustion theory, mathematical biology, the theory of partial differential equations. Hence, there have been many published papers that are devoted to the existence of solutions of boundary value problems for the p-Laplacian operator equations, see $[9,22,23,34,35,41]$ and their references. On the other hand, it has been noticed that most of the above-mentioned work on the topic is based on Riemann-Liouville or Caputo fractional derivatives. In 1892, Hadamard [27] introduced another fractional derivative, which differs from the above-mentioned ones because its definition involves the logarithmic function of arbitrary exponent and named the Hadamard derivative. For some developments on the $\mathrm{f}$ of the Hadamard fractional differential equations, we can refer to $[15,17,25,43]$. Very recently Jarad et al [30] have modified the Hadamard fractional derivative into a more suitable one having physical interpretable initial conditions similar to the singles in the Caputo setting and called it Caputo-Hadamard fractional derivative. Details and properties of the modified derivative can be found in [30]. To the best of our knowledge, few results can be found in the literature concerning boundary value problems for Caputo-Hadamard fractional differential equations $[2,7,8,16]$. There are no contributions, as far as we know, concerning the Caputo-Hadamard fractional differential equations with p-Laplacian operator in Banach spaces. As a result, the goal of this paper is to enrich this academic area. So, in this paper, we mainly study the following problem of Caputo-Hadamard fractional differential equation with p-Laplacian operator of the form

$$
{ }_{H}^{C} \mathcal{D}_{1}^{\beta}\left(\phi_{p}\left[{ }_{H}^{C} \mathcal{D}_{1}^{\alpha} u(t)\right]\right)=f(t, u(t)), \quad 1<\alpha \leq 2 t \in J:=[1, T],
$$

supplemented with boundary conditions

$$
\begin{aligned}
a_{1} u(1)+b_{1}{ }_{H}^{C} \mathcal{D}_{1}^{\gamma} u(1) & =\lambda_{1}{ }^{{ }^{H}}{ }_{\mathcal{I}_{1}^{\sigma_{1}}} u\left(\eta_{1}\right), 0<\gamma \leq 1 \\
a_{2} u(T)+b_{2}{ }_{H} \mathcal{D}_{1}^{\gamma} u(T) & =\lambda_{2}{ }^{{ }^{H}} \mathcal{I}_{1}^{\sigma_{2}} u\left(\eta_{2}\right), 1<\eta_{1}, \eta_{2}<T \\
{ }_{H}^{C} \mathcal{D}_{1}^{\alpha} u(1) & =0,
\end{aligned}
$$

where ${ }_{H}^{C} \mathcal{D}_{1}^{\mu}$ is the Caputo-Hadamard fractional derivative order $\mu \in\{\alpha, \beta, \gamma\}$ such that $1<\alpha \leq 2,0<\beta, \gamma \leq 1,{ }^{H} \mathcal{I}_{1}^{\theta}$ is the Hadamard fractional integral of order $\theta>0, \theta \in\left\{\sigma_{1}, \sigma_{2}\right\}$ and $f:[1, T] \times E \longrightarrow E$ is a given function satisfying some 
assumptions that will be specified later, $E$ is a Banach space with norm $\|\cdot\|$. $a_{i}, b_{i}, \lambda_{i}, i=1,2$ are suitably chosen real constants.

This paper is organized as follows. The second section provides the definitions and preliminary results to be used in this paper. The existence results, which rely on Mönch's fixed point theorem have been presented in Section 3. An example illustrating the obtained results is presented in Section 4, and the paper concludes with some conclusions in Section 5 .

\section{Preliminaries}

We start this section by introducing some necessary definitions and basic results required for further developments.

Let $C(J, E)$ be the Banach space of all continuous functions $u$ from $J$ into $E$ with the supremum (uniform) norm

$$
\|u\|_{\infty}=\sup \{\|u(t)\|, t \in J\} .
$$

By $L^{1}(J)$ we denote the space of Bochner-integrable functions $u: J \rightarrow E$, with the norm

$$
\|u\|_{1}=\int_{1}^{T}\|x(t)\| \mathrm{dt} .
$$

Next, we define the Kuratowski measure of noncompactness and give some of its important properties.

Definition 2.1. [10] Let $E$ be a Banach space, $\Omega_{E}$ the bounded subsets of $E$ The Kuratowski measure of noncompactness is the map $\kappa: \Omega_{E} \rightarrow[0, \infty)$ defined by

$$
\kappa(B)=\inf \left\{\varepsilon>0: B \subset \cup_{j=1}^{m} B_{j}, B \in \Omega_{E} \text { and } \operatorname{diam}\left(B_{j}\right) \leq \varepsilon\right\} .
$$

Properties 2.1. The Kuratowski measure of noncompactness satisfies some properties. For more details see [10].

(1) $A \subset B \Rightarrow \kappa(A) \leq \kappa(B)$,

(2) $\kappa(A)=0 \Leftrightarrow A$ is relatively compact,

(3) $\kappa(A)=\kappa(\bar{A})=\kappa(\operatorname{conv}(A))$, where $\bar{A}$ and conv $A$ represent the closure and the convex hull of $A$, respectively,

(4) $\kappa(A+B) \leq \kappa(A)+\kappa(B)$,

(5) $\kappa(\lambda A)=|\lambda| \kappa(A), \lambda \in \mathbb{R}$.

Now, we give some results and properties from the theory of of fractional calculus. We begin by defining Hadamard fractional integrals and derivatives. In what follows, 
Definition 2.2. [33] The Hadamard fractional integral of order $\alpha>0$, for a function $u \in L^{1}(J)$, is defined as

$$
\left({ }^{H} \mathcal{I}_{1}^{\alpha} u\right)(t)=\frac{1}{\Gamma(\alpha)} \int_{1}^{t}\left(\log \frac{t}{s}\right)^{\alpha-1} u(s) \frac{\mathrm{d} s}{s}, \quad \alpha>0
$$

where $\Gamma(\cdot)$ is the (Euler's) Gamma function

$$
\Gamma(\alpha)=\int_{0}^{+\infty} e^{-t} t^{\alpha-1} \mathrm{dt}, \quad \alpha>0 .
$$

Set

$$
\delta=t \frac{\mathrm{d}}{\mathrm{dt}}, \quad \alpha>0, n=[\alpha]+1,
$$

where $[\alpha]$ denotes the integer part of $\alpha$. Define the space

$$
A C_{\delta}^{n}[1, T]:=\left\{u:[1, T] \longrightarrow \mathbb{R}: \delta^{n-1} u(t) \in A C([1, T])\right\}
$$

Definition 2.3. [33] The Hadamard fractional derivative of order $\alpha>0$ applied to the function $u \in A C_{\delta}^{n}[1, T]$ is defined as

$$
\left({ }^{H} \mathcal{D}_{1}^{\alpha} u\right)(t)=\delta^{n}\left({ }^{H} \mathcal{I}_{1}^{n-\alpha} u\right)(t)
$$

Definition 2.4. $[30,33]$ The Caputo-Hadamard fractional derivative of order $\alpha>$ 0 applied to the function $u \in A C_{\delta}^{n}[1, T]$ is defined as

$$
\left({ }_{H}^{C} \mathcal{D}_{1}^{\alpha} u\right)(t)=\left({ }^{H} \mathcal{I}_{1}^{n-\alpha} \delta^{n} u\right)(t)
$$

Lemmas of the following type are rather standard in the study of fractional differential equations.

Lemma 2.1. [30, 33] Let $\alpha>0, r>0, n=[\alpha]+1$, and $a>0$, then the following relations hold

- $\left({ }^{H} \mathcal{I}_{1}^{\alpha}\left(\log \frac{s}{a}\right)^{r-1}\right)(t)=\frac{\Gamma(r)}{\Gamma(\alpha+r)}\left(\log \frac{t}{a}\right)^{\alpha+r-1}$,

$$
\begin{aligned}
& \left({ }_{H}^{C} \mathcal{D}_{1}^{\alpha}\left(\log \frac{s}{a}\right)^{r-1}\right)(t)=\frac{\Gamma(r)}{\Gamma(r-\alpha)}\left(\log \frac{t}{a}\right)^{r-\alpha-1}, \quad(r>n), \\
& \left({ }_{H}^{C} \mathcal{D}_{1}^{\alpha}\left(\log \frac{s}{a}\right)^{r-1}\right)(t)=0, \quad r \in\{0, \ldots, n-1\} .
\end{aligned}
$$

Lemma 2.2. $[24,33]$ Let $\alpha>\beta>0$, and $u \in A C_{\delta}^{n}[1, T]$. Then we have:

- ${ }^{H} \mathcal{I}_{1}^{\alpha}{ }^{H} \mathcal{I}_{1}^{\beta} u(t)={ }^{H} \mathcal{I}_{1}^{\alpha+\beta} u(t)$ 
- ${ }_{H}^{C} \mathcal{D}_{1}^{\alpha}{ }^{H} \mathcal{I}_{1}^{\alpha} u(t)=u(t)$

- ${ }_{H}^{C} \mathcal{D}_{1}^{\beta}{ }^{H} \mathcal{I}_{1}^{\alpha} u(t)={ }^{H} \mathcal{I}_{1}^{\alpha-\beta} u(t)$.

Lemma 2.3. [30,33] Let $\alpha \geq 0$, and $n=[\alpha]+1$. If $u \in A C_{\delta}^{n}[1, T]$, then the Caputo-Hadamard fractional differential equation

$$
\left({ }_{H}^{C} \mathcal{D}_{1}^{\alpha} u\right)(t)=0
$$

has a solution:

$$
u(t)=\sum_{j=0}^{n-1} c_{j}(\log t)^{j},
$$

and the following formula holds:

$$
{ }^{H} \mathcal{I}_{1}^{\alpha}\left({ }_{H}^{C} \mathcal{D}_{1}^{\alpha} u(t)\right)=u(t)+\sum_{j=0}^{n-1} c_{j}(\log t)^{j},
$$

where $c_{j} \in \mathbb{R}, j=0,1,2, \ldots, n-1$.

Remark 2.1. Note that for an abstract function $u: J \longrightarrow E$, the integrals which appear in the previous definitions are taken in Bochner's sense. (see, for instance, [40]).

In the sequel we will make use of the following fixed point theorem.

Theorem 2.2. (Mönch's fixed point theorem [36]). Let D be a bounded, closed and convex subset of a Banach space such that $0 \in D$, and let $\mathcal{N}$ be a continuous mapping of $D$ into itself. If the implication

$$
V=\overline{\operatorname{conv}} \mathcal{N}(V), \text { or } V=\mathcal{N}(V) \cup\{0\} \Rightarrow \kappa(V)=0,
$$

holds for every subset $V \subset D$, then $\mathcal{N}$ has a fixed point.

Lemma 2.4. [6] Let $H \subset C(J, E)$ be a bounded and equicontinuous subset. Then the function $t \rightarrow \kappa(H(t))$ is continuous on $J$, and

$$
\kappa_{C}(H)=\max _{t \in J} \kappa(H(t))
$$

and

$$
\kappa\left(\int_{J} u(s) \mathrm{ds}\right) \leq \int_{J} \kappa(H(s)) \mathrm{ds},
$$

where $H(s)=\{u(s): u \in H, s \in J\}$, and $\kappa_{C}$ is the Kuratowski measure of noncompactness defined on the bounded sets of $C(J, E)$.

Definition 2.5. [45] A function $f:[1, T] \times E \longrightarrow E$ is said to satisfy the Carathéodory conditions, if the following hold

- $f(t, u)$ is measurable with respect to $t$ for $u \in E$,

- $f(t, u)$ is continuous with respect to $u \in E$ for a.e. $t \in J$. 


\section{Main Results}

Before starting and proving our main result, we have introduced the following auxiliary lemma.

Lemma 3.1. For a given $h \in C(J, \mathbb{R})$, the unique solution of the linear fractional boundary value problem

$$
{ }_{H}^{C} \mathcal{D}_{1}^{\alpha} u(t)=h(t), 1<\alpha \leq 2 t \in J:=[1, T],
$$

supplemented with boundary conditions

$$
\begin{aligned}
a_{1} u(1)+b_{1}{ }_{H}^{C} \mathcal{D}_{1}^{\gamma} u(1) & =\lambda_{1}{ }^{{ }^{H}} \mathcal{I}_{1}^{\sigma_{1}} u\left(\eta_{1}\right), 0<\gamma \leq 1 \\
a_{2} u(T)+b_{2}{ }_{H} \mathcal{D}_{1}^{\gamma} u(T) & =\lambda_{2}{ }^{{ }^{H}} \mathcal{I}_{1}^{\sigma_{2}} u\left(\eta_{2}\right), 1<\eta_{1}, \eta_{2}<T,
\end{aligned}
$$

is given by

$$
\begin{aligned}
u(t) & ={ }^{H} \mathcal{I}_{1}^{\alpha} h(t)+\mu_{1}(t){ }^{H} \mathcal{I}_{1}^{\sigma_{1}+\alpha} h\left(\eta_{1}\right)+\mu_{2}(t)\left(\lambda_{2}{ }^{H} \mathcal{I}_{1}^{\sigma_{2}+\alpha} h\left(\eta_{2}\right)\right. \\
& \left.-\left(a_{2}{ }^{H} \mathcal{I}_{1}^{\alpha} h(T)+b_{2}{ }^{H} \mathcal{I}_{1}^{\alpha-\gamma} h(T)\right)\right),
\end{aligned}
$$

where

$$
\begin{aligned}
\mu_{1}(t) & =\lambda_{1}\left(\Delta_{1}-\Delta_{2} \log t\right), \quad \mu_{2}(t)=\lambda_{1} \Delta_{3}+\Delta_{4} \log t \\
\Delta_{1} & =\frac{1}{\Delta}\left(a_{2} \log T+\frac{b_{2}(\log T)^{1-\gamma}}{\Gamma(2-\gamma)}-\frac{\lambda_{2}\left(\log \eta_{2}\right)^{\sigma_{2}+1}}{\Gamma\left(\sigma_{2}+2\right)}\right) \\
\Delta_{2} & =\frac{1}{\Delta}\left(a_{2} \log T-\frac{\lambda_{2}\left(\log \eta_{2}\right)^{\sigma_{2}}}{\Gamma\left(\sigma_{2}+1\right)}\right) \\
(3.4) \Delta_{3} & =\frac{\lambda_{1}\left(\log \eta_{1}\right)^{\sigma_{1}+1}}{\Delta \Gamma\left(\sigma_{1}+2\right)} ; \\
\Delta_{4} & =\frac{1}{\Delta}\left(a_{1}-\frac{\lambda_{1}\left(\log \eta_{1}\right)^{\sigma_{1}}}{\Gamma\left(\sigma_{1}+1\right)}\right) \\
\Delta & =\left(a_{1}-\frac{\lambda_{1}\left(\log \eta_{1}\right)^{\sigma_{1}}}{\Gamma\left(\sigma_{1}+1\right)}\right)\left(a_{2} \log T+\frac{b_{2}(\log T)^{1-\gamma}}{\Gamma(2-\gamma)}-\frac{\lambda_{2}\left(\log \eta_{2}\right)^{\sigma_{2}+1}}{\Gamma\left(\sigma_{2}+2\right)}\right) \\
& +\frac{\lambda_{1}\left(\log \eta_{1}\right)^{\sigma_{1}+1}}{\Gamma\left(\sigma_{1}+2\right)}\left(a_{2} \log T-\frac{\lambda_{2}\left(\log \eta_{2}\right)^{\sigma_{2}}}{\Gamma\left(\sigma_{2}+1\right)}\right) \neq 0 .
\end{aligned}
$$

Proof. By applying Lemma 2.3, we may reduce (3.1) to an equivalent integral equation

$$
u(t)={ }^{H} \mathcal{I}_{1}^{\alpha} h(t)+k_{0}+k_{1} \log (t), \quad k_{0}, k_{1} \in \mathbb{R} .
$$

Applying the boundary conditions (3.2) in (3.5) we may obtain

$$
\begin{aligned}
& { }^{H_{\mathcal{I}}}{ }_{1}^{\sigma_{i}} u\left(\eta_{i}\right)={ }^{H} \mathcal{I}_{1}^{\sigma_{i}+\alpha} h\left(\eta_{i}\right)+k_{0} \frac{\left(\log \eta_{i}\right)^{\sigma_{i}}}{\Gamma\left(\sigma_{i}+1\right)}+k_{1} \frac{\left(\log \eta_{i}\right)^{\sigma_{i}+1}}{\Gamma\left(\sigma_{i}+2\right)}, i=1,2 . \\
& { }_{H}^{C} \mathcal{D}_{1}^{\gamma} u(T)={ }^{H} \mathcal{I}_{1}^{\alpha-\gamma} h(T)+k_{1} \frac{\Gamma(2)}{\Gamma(2-\gamma)}(\log T)^{1-\gamma} .
\end{aligned}
$$


After collecting the similar terms in one part, we have the following equations:

$$
\begin{aligned}
\left(a_{1}-\frac{\lambda_{1}\left(\log \eta_{1}\right)^{\sigma_{1}}}{\Gamma\left(\sigma_{1}+1\right)}\right) k_{0} & -\frac{\lambda_{1}\left(\log \eta_{1}\right)^{\sigma_{1}+1}}{\Gamma\left(\sigma_{1}+2\right)} k_{1}=\lambda_{1}{ }^{{ }^{H}} \mathcal{I}_{1}^{\sigma_{1}+\alpha} h\left(\eta_{1}\right), \\
\left(a_{2} \log T-\frac{\lambda_{2}\left(\log \eta_{2}\right)^{\sigma_{2}}}{\Gamma\left(\sigma_{2}+1\right)}\right) k_{0} & +\left(a_{2} \log T+\frac{b_{2}(\log T)^{1-\gamma}}{\Gamma(2-\gamma)}-\frac{\lambda_{2}\left(\log \eta_{2}\right)^{\sigma_{2}+1}}{\Gamma\left(\sigma_{2}+2\right)}\right) k_{1} \\
& =\lambda_{2}{ }^{{ }^{H}} \mathcal{I}_{1}^{\sigma_{2}+\alpha} h\left(\eta_{2}\right)-a_{2}{ }^{H} \mathcal{I}_{1}^{\alpha} h(T)-b_{2}{ }^{H} \mathcal{I}_{1}^{\alpha-\gamma} h(T) .
\end{aligned}
$$

Therefore, we get

$$
\begin{aligned}
k_{0} & =\frac{\lambda_{1}}{\Delta}\left(a_{2} \log T+\frac{b_{2}(\log T)^{1-\gamma}}{\Gamma(2-\gamma)}-\frac{\lambda_{2}\left(\log \eta_{2}\right)^{\sigma_{2}+1}}{\Gamma\left(\sigma_{2}+2\right)}\right){ }^{{ }^{H}} \mathcal{I}_{1}^{\alpha+\sigma_{1}} h\left(\eta_{1}\right) \\
& +\frac{\lambda_{1}\left(\log \eta_{1}\right)^{\sigma_{1}+1}}{\Delta \Gamma\left(\sigma_{1}+2\right)}\left(\lambda_{2}{ }^{H} \mathcal{I}_{1}^{\sigma_{2}+\alpha} h\left(\eta_{2}\right)-\left(a_{2}{ }^{H} \mathcal{I}_{1}^{\alpha} h(T)+b_{2}{ }^{{ }^{H}} \mathcal{I}_{1}^{\alpha-\gamma} h(T)\right)\right), \\
k_{1} & =\frac{\lambda_{1}}{\Delta}\left(a_{1}-\frac{\lambda_{1}\left(\log \eta_{1}\right)^{\sigma_{1}}}{\Gamma\left(\sigma_{1}+1\right)}\right)\left(\lambda_{2} I_{0^{+}}^{\sigma_{2}+\alpha} h\left(\eta_{2}\right)-\left(a_{2}{ }^{H} \mathcal{I}_{1}^{\alpha} h(T)+b_{2}{ }^{H} \mathcal{I}_{1}^{\alpha-\gamma} h(1)\right)\right), \\
& -\frac{\lambda_{1}}{\Delta}\left(a_{2} \log T-\frac{\lambda_{2}\left(\log \eta_{2}\right)^{\sigma_{2}}}{\Gamma\left(\sigma_{2}+1\right)}\right) I_{0^{+}}^{\sigma_{1}+\alpha} h\left(\eta_{1}\right) .
\end{aligned}
$$

Substituting the value of $k_{0}, k_{1}$ in (3.5) we get (3.3), which completes the proof.

Lemma 3.2. Let $1<\alpha \leq 2,0<\beta \leq 1$. Then the boundary value problem of the fractional differential equation

$$
{ }_{H}^{C} \mathcal{D}_{1}^{\beta}\left(\phi_{p}\left[{ }_{H}^{C} \mathcal{D}_{1}^{\alpha} u(t)\right]\right)=f(t, u(t)), \quad 1<\alpha \leq 2 t \in J:=[1, T],
$$

supplemented with boundary conditions

$$
\begin{aligned}
a_{1} u(1)+b_{1}{ }_{H}^{C} \mathcal{D}_{1}^{\gamma} u(1) & =\lambda_{1}{ }^{H} \mathcal{I}_{1}^{\sigma_{1}} u\left(\eta_{1}\right), 0<\gamma \leq 1 \\
a_{2} u(T)+b_{2}{ }_{H}^{C} \mathcal{D}_{1}^{\gamma} u(T) & =\lambda_{2}{ }^{{ }^{H}} \mathcal{I}_{1}^{\sigma_{2}} u\left(\eta_{2}\right), 1<\eta_{1}, \eta_{2}<T \\
{ }_{H}^{C} \mathcal{D}_{1}^{\alpha} u(1) & =0,
\end{aligned}
$$

has a unique solution

$$
\begin{aligned}
u(t) & =\frac{1}{\Gamma(\alpha)} \int_{1}^{t}\left(\log \frac{t}{s}\right)^{\alpha-1} \phi_{q}\left(\int_{1}^{s} \frac{\left(\log \frac{s}{\tau}\right)^{\beta-1}}{\Gamma(\beta)} f(\tau, u(\tau)) \frac{\mathrm{d} \tau}{\tau}\right) \frac{\mathrm{d} s}{\mathrm{~s}} \\
& +\frac{\mu_{1}(t)}{\Gamma\left(\sigma_{1}+\alpha\right)} \int_{1}^{\eta_{1}}\left(\log \frac{\eta_{1}}{s}\right)^{\sigma_{1}+\alpha-1} \phi_{q}\left(\int_{1}^{s} \frac{\left(\log \frac{s}{\tau}\right)^{\beta-1}}{\Gamma(\beta)} f(\tau, u(\tau)) \frac{\mathrm{d} \tau}{\tau}\right) \frac{\mathrm{d} s}{\mathrm{~s}} \\
& +\frac{\lambda_{2} \mu_{2}(t)}{\Gamma\left(\sigma_{2}+\alpha\right)} \int_{1}^{\eta_{2}}\left(\log \frac{\eta_{2}}{s}\right)^{\sigma_{2}+\alpha-1} \phi_{q}\left(\int_{1}^{s} \frac{\left(\log \frac{s}{\tau}\right)^{\beta-1}}{\Gamma(\beta)} f(\tau, u(\tau)) \frac{\mathrm{d} \tau}{\tau}\right) \frac{\mathrm{d} s}{\mathrm{~s}}
\end{aligned}
$$




$$
\begin{aligned}
& -\frac{a_{2} \mu_{2}(t)}{\Gamma(\alpha)} \int_{1}^{T}\left(\log \frac{T}{s}\right)^{\alpha-1} \phi_{q}\left(\int_{1}^{s} \frac{\left(\log \frac{s}{\tau}\right)^{\beta-1}}{\Gamma(\beta)} f(\tau, u(\tau)) \frac{\mathrm{d} \tau}{\tau}\right) \frac{\mathrm{ds}}{\mathrm{s}} \\
& -\frac{b_{2} \mu_{2}(t)}{\Gamma(\alpha-\gamma)} \int_{1}^{T}\left(\log \frac{T}{s}\right)^{\alpha-\gamma-1} \phi_{q}\left(\int_{1}^{s} \frac{\left(\log \frac{s}{\tau}\right)^{\beta-1}}{\Gamma(\beta)} f(\tau, u(\tau)) \frac{\mathrm{d} \tau}{\tau}\right) \frac{\mathrm{ds}}{\mathrm{s}}
\end{aligned}
$$

Proof. From Lemma 2.3 and the boundary value problem (3.6)-(3.7), we have

$$
\begin{aligned}
{ }^{H} \mathcal{I}_{1 H}^{\beta}{ }_{\mathcal{H}} \mathcal{D}_{1}^{\beta}\left(\phi_{p}\left[{ }_{H}^{C} \mathcal{D}_{1}^{\alpha} u(t)\right]\right) & ={ }^{H} \mathcal{I}_{1}^{\beta} f(t, u(t)) \\
& =\phi_{p}\left[{ }_{H}^{C} \mathcal{D}_{1}^{\alpha} u(t)\right]+d_{0}, \quad d_{0} \in \mathbb{R},
\end{aligned}
$$

that is

$$
\phi_{p}\left[{ }_{H}^{C} \mathcal{D}_{1}^{\alpha} u(t)\right]={ }^{{ }^{H}} \mathcal{I}_{1}^{\beta} f(t, u(t))-d_{0}, \quad d_{0} \in \mathbb{R},
$$

By ${ }_{H}^{C} \mathcal{D}_{1}^{\alpha} u(1)=0$, we have $d_{0}=0$. So,

$$
{ }_{H}^{C} \mathcal{D}_{1}^{\alpha} u(t)=\phi_{q}\left[{ }^{H} \mathcal{I}_{1}^{\beta} f(t, u(t))\right] .
$$

Thus, the boundary value problem (3.6)-(3.7) is equivalent to the following problem:

$$
\begin{gathered}
{ }_{H}^{C} \mathcal{D}_{1}^{\alpha} u(t)=\phi_{q}\left[{ }^{H} \mathcal{I}_{1}^{\beta} f(t, u(t))\right], t \in J:=[1, T], \\
a_{1} u(1)+b_{1}{ }_{H}^{C} \mathcal{D}_{1}^{\gamma} u(1)=\lambda_{1}{ }^{H^{\prime}} \mathcal{I}_{1}^{\sigma_{1}} u\left(\eta_{1}\right), 0<\gamma \leq 1 \\
a_{2} u(T)+b_{2}{ }_{H}^{C} \mathcal{D}_{1}^{\gamma} u(T)=\lambda_{2}{ }^{H^{1}} \mathcal{I}_{1}^{\sigma_{2}} u\left(\eta_{2}\right), 1<\eta_{1}, \eta_{2}<T .
\end{gathered}
$$

Lemma 3.1 implies that boundary value problem (3.6)-(3.7) has a unique solution,

$$
\begin{aligned}
u(t) & =\frac{1}{\Gamma(\alpha)} \int_{1}^{t}\left(\log \frac{t}{s}\right)^{\alpha-1} \phi_{q}\left(\int_{1}^{s} \frac{\left(\log \frac{s}{\tau}\right)^{\beta-1}}{\Gamma(\beta)} f(\tau, u(\tau)) \frac{\mathrm{d} \tau}{\tau}\right) \frac{\mathrm{d} s}{\mathrm{~s}} \\
& +\frac{\mu_{1}(t)}{\Gamma\left(\sigma_{1}+\alpha\right)} \int_{1}^{\eta_{1}}\left(\log \frac{\eta_{1}}{s}\right)^{\sigma_{1}+\alpha-1} \phi_{q}\left(\int_{1}^{s} \frac{\left(\log \frac{s}{\tau}\right)^{\beta-1}}{\Gamma(\beta)} f(\tau, u(\tau)) \frac{\mathrm{d} \tau}{\tau}\right) \frac{\mathrm{d} s}{\mathrm{~s}} \\
& +\frac{\lambda_{2} \mu_{2}(t)}{\Gamma\left(\sigma_{2}+\alpha\right)} \int_{1}^{\eta_{2}}\left(\log \frac{\eta_{2}}{s}\right)^{\sigma_{2}+\alpha-1} \phi_{q}\left(\int_{1}^{s} \frac{\left(\log \frac{s}{\tau}\right)^{\beta-1}}{\Gamma(\beta)} f(\tau, u(\tau)) \frac{\mathrm{d} \tau}{\tau}\right) \frac{\mathrm{d} s}{\mathrm{~s}} \\
& -\frac{a_{2} \mu_{2}(t)}{\Gamma(\alpha)} \int_{1}^{T}\left(\log \frac{T}{s}\right)^{\alpha-1} \phi_{q}\left(\int_{1}^{s} \frac{\left(\log \frac{s}{\tau}\right)^{\beta-1}}{\Gamma(\beta)} f(\tau, u(\tau)) \frac{\mathrm{d} \tau}{\tau}\right) \frac{\mathrm{d} s}{\mathrm{~s}} \\
& -\frac{b_{2} \mu_{2}(t)}{\Gamma(\alpha-\gamma)} \int_{1}^{T}\left(\log \frac{T}{s}\right)^{\alpha-\gamma-1} \phi_{q}\left(\int_{1}^{s} \frac{\left(\log \frac{s}{\tau}\right)^{\beta-1}}{\Gamma(\beta)} f(\tau, u(\tau)) \frac{\mathrm{d} \tau}{\tau}\right) \frac{\mathrm{d} s}{\mathrm{~s}} .
\end{aligned}
$$

This completes the proof. 
In the following, for computational convenience we put

$$
\begin{aligned}
\mathcal{M}_{f} & =\frac{\left\|p_{f}\right\| \Gamma(\beta(q-1)+1)}{\Gamma(\beta+1)^{q-1}}\left\{\frac{(\log T)^{\alpha+\beta(q-1)}\left(1+\left|a_{2}\right| \tilde{\mu_{2}}\right)}{\Gamma(\alpha+\beta(q-1)+1)}+\frac{\tilde{\mu}_{1}\left(\log \eta_{1}\right)^{\sigma_{1}+\alpha+\beta(q-1)}}{\Gamma\left(\sigma_{1}+\alpha+\beta(q-1)+1\right)}\right. \\
& \left.+\tilde{\mu_{2}}\left[\frac{\left|\lambda_{2}\right|\left(\log \eta_{2}\right)^{\sigma_{2}+\alpha+\beta(q-1)}}{\Gamma\left(\sigma_{2}+\alpha+\beta(q-1)+1\right)}+\frac{\left|b_{2}\right|(\log T)^{\alpha-\gamma+\beta(q-1)}}{\Gamma(\alpha-\gamma+\beta(q-1)+1)}\right]\right\},
\end{aligned}
$$

where $\tilde{\mu_{1}}=\left|\lambda_{1}\right|\left(\left|\Delta_{1}\right|+\left|\Delta_{2}\right| \log T\right), \tilde{\mu_{2}}=\left|\lambda_{1} \Delta_{3}\right|+\left|\Delta_{4}\right| \log T$,

$$
\begin{aligned}
\mathcal{L}_{f} & =\frac{\left\|p_{f}\right\| \Gamma(\beta(q-1)+1)}{\Gamma(\beta+1)^{q-1}}\left\{\frac{(\log T)^{\alpha+\beta(q-1)-1}}{\Gamma(\alpha+\beta(q-1))}+\frac{\left|\lambda_{1} \Delta_{2}\right|\left(\log \eta_{1}\right)^{\sigma_{1}+\alpha+\beta(q-1)}}{T \Gamma\left(\sigma_{1}+\alpha+\beta(q-1)+1\right)}\right. \\
& +\frac{\left|\Delta_{4}\right|}{T}\left[\frac{\left|\lambda_{2}\right|\left(\log \eta_{2}\right)^{\sigma_{2}+\alpha+\beta(q-1)}}{\Gamma\left(\sigma_{2}+\alpha+\beta(q-1)+1\right)}+\frac{\left|a_{2}\right|(\log T)^{\alpha+\beta(q-1)}}{\Gamma(\alpha+\beta(q-1)+1)}\right. \\
(3.10)+ & \left.\left.\frac{\left|b_{2}\right|(\log T)^{\alpha-\gamma+\beta(q-1)}}{\Gamma(\alpha-\gamma+\beta(q-1)+1)}\right]\right\}
\end{aligned}
$$

Now, we shall prove the following theorem concerning the existence of solutions of problem (1.1)-(1.2)

Theorem 3.1. Assume that the following hypotheses hold:

(H1) The function $f:[1, T] \times E \longrightarrow E$ satisfies Carathéodory conditions

(H2) There exisst $p_{f} \in L^{\infty}\left(J, \mathbb{R}_{+}\right)$and a continuous nondecreasing function $\psi$ : $\mathbb{R}_{+} \longrightarrow \mathbb{R}_{+}$such that

$$
\|f(t, u)\| \leq \phi_{p}\left(p_{f}(t) \psi(\|u\|)\right) \text { for a.e. } t \in J \text { and each } u \in E \text {. }
$$

(H3) For each bounded set $D \subset E$, and each $t \in J$, the following inequality holds

$$
\kappa(f(t, D)) \leq \phi_{p}\left(p_{f}(t) \kappa(D)\right)
$$

If

$$
\mathcal{M}_{f}<1
$$

then the problem (1.1)-(1.2) has at least one solution defined on J.

Proof. Consider the operator $\mathcal{N}: C(J, E) \longrightarrow C(J, E)$ defined by:

$$
\mathcal{N} u(t)=\frac{1}{\Gamma(\alpha)} \int_{1}^{t}\left(\log \frac{t}{s}\right)^{\alpha-1} \phi_{q}\left(\int_{1}^{s} \frac{\left(\log \frac{s}{\tau}\right)^{\beta-1}}{\Gamma(\beta)} f(\tau, u(\tau)) \frac{\mathrm{d} \tau}{\tau}\right) \frac{\mathrm{d} s}{\mathrm{~s}}
$$




$$
\begin{aligned}
& +\frac{\mu_{1}(t)}{\Gamma\left(\sigma_{1}+\alpha\right)} \int_{1}^{\eta_{1}}\left(\log \frac{\eta_{1}}{s}\right)^{\sigma_{1}+\alpha-1} \phi_{q}\left(\int_{1}^{s} \frac{\left(\log \frac{s}{\tau}\right)^{\beta-1}}{\Gamma(\beta)} f(\tau, u(\tau)) \frac{\mathrm{d} \tau}{\tau}\right) \frac{\mathrm{d} s}{\mathrm{~s}} \\
& +\frac{\lambda_{2} \mu_{2}(t)}{\Gamma\left(\sigma_{2}+\alpha\right)} \int_{1}^{\eta_{2}}\left(\log \frac{\eta_{2}}{s}\right)^{\sigma_{2}+\alpha-1} \phi_{q}\left(\int_{1}^{s} \frac{\left(\log \frac{s}{\tau}\right)^{\beta-1}}{\Gamma(\beta)} f(\tau, u(\tau)) \frac{\mathrm{d} \tau}{\tau}\right) \frac{\mathrm{d} s}{\mathrm{~s}} \\
& -\frac{a_{2} \mu_{2}(t)}{\Gamma(\alpha)} \int_{1}^{T}\left(\log \frac{T}{s}\right)^{\alpha-1} \phi_{q}\left(\int_{1}^{s} \frac{\left(\log \frac{s}{\tau}\right)^{\beta-1}}{\Gamma(\beta)} f(\tau, u(\tau)) \frac{\mathrm{d} \tau}{\tau}\right) \frac{\mathrm{d} s}{\mathrm{~s}} \\
& -\frac{b_{2} \mu_{2}(t)}{\Gamma(\alpha-\gamma)} \int_{1}^{T}\left(\log \frac{T}{s}\right)^{\alpha-\gamma-1} \phi_{q}\left(\int_{1}^{s} \frac{\left(\log \frac{s}{\tau}\right)^{\beta-1}}{\Gamma(\beta)} f(\tau, u(\tau)) \frac{\mathrm{d} \tau}{\tau}\right) \frac{\mathrm{d} s}{\mathrm{~s}}
\end{aligned}
$$

From Lemma 3.2, the fixed points of the operator $\mathcal{N}$ are solution of the problem (1.1)-(1.2).

Let $R>0$, such that

$$
R \geq \mathcal{M}_{f} \psi(R)
$$

and consider the ball

$$
B_{R}=\left\{w \in C(J, E):\|w\|_{\infty} \leq R\right\} .
$$

We shall show that the operator $\mathcal{N}$ satisfies all the assumptions of Theorem 2.2. Take $u \in B_{R}, t \in J$ we have

$$
\begin{gathered}
\leq \mathcal{N} u(t) \| \\
\leq \frac{1}{\Gamma(\alpha)} \int_{1}^{t}\left(\log \frac{t}{s}\right)^{\alpha-1} \phi_{q}\left(\int_{1}^{s} \frac{\left(\log \frac{s}{\tau}\right)^{\beta-1}}{\Gamma(\beta)}\|f(\tau, u(\tau))\| \frac{\mathrm{d} \tau}{\tau}\right) \frac{\mathrm{d} \mathrm{s}}{\mathrm{s}} \\
+\frac{\left|\mu_{1}(t)\right|}{\Gamma\left(\sigma_{1}+\alpha\right)} \int_{1}^{\eta_{1}}\left(\log \frac{\eta_{1}}{s}\right)^{\sigma_{1}+\alpha-1} \phi_{q}\left(\int_{1}^{s} \frac{\left(\log \frac{s}{\tau}\right)^{\beta-1}}{\Gamma(\beta)}\|f(\tau, u(\tau))\| \frac{\mathrm{d} \tau}{\tau}\right) \frac{\mathrm{d} s}{\mathrm{~s}} \\
+\frac{\left|\lambda_{2} \mu_{2}(t)\right|}{\Gamma\left(\sigma_{2}+\alpha\right)} \int_{1}^{\eta_{2}}\left(\log \frac{\eta_{2}}{s}\right)^{\sigma_{2}+\alpha-1} \phi_{q}\left(\int_{1}^{s} \frac{\left(\log \frac{s}{\tau}\right)^{\beta-1}}{\Gamma(\beta)}\|f(\tau, u(\tau))\| \frac{\mathrm{d} \tau}{\tau}\right) \frac{\mathrm{ds}}{\mathrm{s}} \\
+\frac{\left|a_{2} \mu_{2}(t)\right|}{\Gamma(\alpha)} \int_{1}^{T}\left(\log \frac{T}{s}\right)^{\alpha-1} \phi_{q}\left(\int_{1}^{s} \frac{\left(\log \frac{s}{\tau}\right)^{\beta-1}}{\Gamma(\beta)}\|f(\tau, u(\tau))\| \frac{\mathrm{d} \tau}{\tau}\right) \frac{\mathrm{ds}}{\mathrm{s}} \\
+\frac{\left|b_{2} \mu_{2}(t)\right|}{\Gamma(\alpha-\gamma)} \int_{1}^{T}\left(\log \frac{T}{s}\right)^{\alpha-\gamma-1} \phi_{q}\left(\int_{1}^{s} \frac{\left(\log \frac{s}{\tau}\right)^{\beta-1}}{\Gamma(\beta)}\|f(\tau, u(\tau))\| \frac{d \tau}{\tau}\right) \frac{\mathrm{ds}}{\mathrm{s}}
\end{gathered}
$$

Using hypothese (H2) we get

$\|\mathcal{N} u(t)\|$

$$
\leq \frac{1}{\Gamma(\alpha)} \int_{1}^{t}\left(\log \frac{t}{s}\right)^{\alpha-1} \phi_{q}\left(\int_{1}^{s} \frac{\left(\log \frac{s}{\tau}\right)^{\beta-1}}{\Gamma(\beta)} \phi_{p}\left(p_{f}(\tau) \psi(\|u(\tau)\|)\right) \frac{\mathrm{d} \tau}{\tau}\right) \frac{\mathrm{d} s}{\mathrm{~s}}
$$




$$
\begin{aligned}
& +\frac{\left|\mu_{1}(t)\right|}{\Gamma\left(\sigma_{1}+\alpha\right)} \int_{1}^{\eta_{1}}\left(\log \frac{\eta_{1}}{s}\right)^{\sigma_{1}+\alpha-1} \phi_{q}\left(\int_{1}^{s} \frac{\left(\log \frac{s}{\tau}\right)^{\beta-1}}{\Gamma(\beta)} \phi_{p}\left(p_{f}(\tau) \psi(\|u(\tau)\|)\right) \frac{\mathrm{d} \tau}{\tau}\right) \frac{\mathrm{ds}}{\mathrm{s}} \\
& +\frac{\left|\lambda_{2} \mu_{2}(t)\right|}{\Gamma\left(\sigma_{2}+\alpha\right)} \int_{1}^{\eta_{2}}\left(\log \frac{\eta_{2}}{s}\right)^{\sigma_{2}+\alpha-1} \phi_{q}\left(\int_{1}^{s} \frac{\left(\log \frac{s}{\tau}\right)^{\beta-1}}{\Gamma(\beta)} \phi_{p}\left(p_{f}(\tau) \psi(\|u(\tau)\|)\right) \frac{\mathrm{d} \tau}{\tau}\right) \frac{\mathrm{ds}}{\mathrm{s}} \\
& +\frac{\left|a_{2} \mu_{2}(t)\right|}{\Gamma(\alpha)} \int_{1}^{T}\left(\log \frac{T}{s}\right)^{\alpha-1} \phi_{q}\left(\int_{1}^{s} \frac{\left(\log \frac{s}{\tau}\right)^{\beta-1}}{\Gamma(\beta)} \phi_{p}\left(p_{f}(\tau) \psi(\|u(\tau)\|)\right) \frac{\mathrm{d} \tau}{\tau}\right) \frac{\mathrm{d} s}{\mathrm{~s}} \\
& +\frac{\left|b_{2} \mu_{2}(t)\right|}{\Gamma(\alpha-\gamma)} \int_{1}^{T}\left(\log \frac{T}{s}\right)^{\alpha-\gamma-1} \phi_{q}\left(\int_{1}^{s} \frac{\left(\log \frac{s}{\tau}\right)^{\beta-1}}{\Gamma(\beta)} \phi_{p}\left(p_{f}(\tau) \psi(\|u(\tau)\|)\right) \frac{\mathrm{d} \tau}{\tau}\right) \frac{\mathrm{d} s}{\mathrm{~s}} \\
& \leq \frac{\left\|p_{f}\right\| \psi(\|u\|)}{\Gamma(\alpha)} \int_{1}^{t}\left(\log \frac{t}{s}\right)^{\alpha-1} \phi_{q}\left(\int_{1}^{s} \frac{\left(\log \frac{s}{\tau}\right)^{\beta-1}}{\Gamma(\beta)} \frac{\mathrm{d} \tau}{\tau}\right) \frac{\mathrm{d} s}{\mathrm{~s}} \\
& +\frac{\left|\mu_{1}(t)\right|\left\|p_{f}\right\| \psi(\|u\|)}{\Gamma\left(\sigma_{1}+\alpha\right)} \int_{1}^{\eta_{1}}\left(\log \frac{\eta_{1}}{s}\right)^{\sigma_{1}+\alpha-1} \phi_{q}\left(\int_{1}^{s} \frac{\left(\log \frac{s}{\tau}\right)^{\beta-1}}{\Gamma(\beta)} \frac{\mathrm{d} \tau}{\tau}\right) \frac{\mathrm{ds}}{\mathrm{s}} \\
& +\frac{\mid \lambda_{2} \mu_{2}(t)\left\|p_{f}\right\| \psi(\|u\|)}{\Gamma\left(\sigma_{2}+\alpha\right)} \int_{1}^{\eta_{2}}\left(\log \frac{\eta_{2}}{s}\right)^{\sigma_{2}+\alpha-1} \phi_{q}\left(\int_{1}^{s} \frac{\left(\log \frac{s}{\tau}\right)^{\beta-1}}{\Gamma(\beta)} \frac{\mathrm{d} \tau}{\tau}\right) \frac{\mathrm{d} s}{\mathrm{~s}} \\
& +\frac{\left|a_{2} \mu_{2}(t)\right|\left\|p_{f}\right\| \psi(\|u\|)}{\Gamma(\alpha)} \int_{1}^{T}\left(\log \frac{T}{s}\right)^{\alpha-1} \phi_{q}\left(\int_{1}^{s} \frac{\left(\log \frac{s}{\tau}\right)^{\beta-1}}{\Gamma(\beta)} \mathrm{d} \tau\right) \frac{\mathrm{d} s}{\mathrm{~s}} \\
& +\frac{\mid b_{2} \mu_{2}(t)\|\| p_{f} \| \psi(\|u\|)}{\Gamma(\alpha-\gamma)} \int_{1}^{T}\left(\log \frac{T}{s}\right)^{\alpha-\gamma-1} \phi_{q}\left(\int_{1}^{s} \frac{\left(\log \frac{s}{\tau}\right)^{\beta-1}}{\Gamma(\beta)} \frac{\mathrm{d} \tau}{\tau}\right) \frac{\mathrm{d} s}{\mathrm{~s}} \\
& =\frac{\left\|p_{f}\right\| \psi(\|u\|)}{\Gamma(\alpha)} \int_{1}^{t}\left(\log \frac{t}{s}\right)^{\alpha-1} \phi_{q}\left(\frac{(\log s)^{\beta}}{\Gamma(\beta+1)}\right) \frac{\mathrm{ds}}{\mathrm{s}} \\
& +\frac{\left|\mu_{1}(t)\right|\left\|p_{f}\right\| \psi(\|u\|)}{\Gamma\left(\sigma_{1}+\alpha\right)} \int_{1}^{\eta_{1}}\left(\log \frac{\eta_{1}}{s}\right)^{\sigma_{1}+\alpha-1} \phi_{q}\left(\frac{(\log s)^{\beta}}{\Gamma(\beta+1)}\right) \frac{\mathrm{ds}}{\mathrm{s}} \\
& +\frac{\left|\lambda_{2} \mu_{2}(t)\right|\left\|p_{f}\right\| \psi(\|u\|)}{\Gamma\left(\sigma_{2}+\alpha\right)} \int_{1}^{\eta_{2}}\left(\log \frac{\eta_{2}}{s}\right)^{\sigma_{2}+\alpha-1} \phi_{q}\left(\frac{(\log s)^{\beta}}{\Gamma(\beta+1)}\right) \frac{\mathrm{ds}}{\mathrm{s}} \\
& +\frac{\left|a_{2} \mu_{2}(t)\right|\left\|p_{f}\right\| \psi(\|u\|)}{\Gamma(\alpha)} \int_{1}^{T}\left(\log \frac{T}{s}\right)^{\alpha-1} \phi_{q}\left(\frac{(\log s)^{\beta}}{\Gamma(\beta+1)}\right) \frac{\mathrm{ds}}{\mathrm{s}} \\
& +\frac{\left|b_{2} \mu_{2}(t)\right|\left\|p_{f}\right\| \psi(\|u\|)}{\Gamma(\alpha-\gamma)} \int_{1}^{T}\left(\log \frac{T}{s}\right)^{\alpha-\gamma-1} \phi_{q}\left(\frac{(\log s)^{\beta}}{\Gamma(\beta+1)}\right) \frac{\mathrm{ds}}{\mathrm{s}} \\
& =\frac{\left\|p_{f}\right\| \psi(\|u\|)}{\Gamma(\alpha)} \int_{1}^{t}\left(\log \frac{t}{s}\right)^{\alpha-1}\left(\frac{(\log s)^{\beta}}{\Gamma(\beta+1)}\right)^{q-1} \frac{\mathrm{ds}}{\mathrm{s}}
\end{aligned}
$$




$$
\begin{aligned}
& +\frac{\left|\mu_{1}(t)\right|\left\|p_{f}\right\| \psi(\|u\|)}{\Gamma\left(\sigma_{1}+\alpha\right)} \int_{1}^{\eta_{1}}\left(\log \frac{\eta_{1}}{s}\right)^{\sigma_{1}+\alpha-1}\left(\frac{(\log s)^{\beta}}{\Gamma(\beta+1)}\right)^{q-1} \frac{\mathrm{ds}}{\mathrm{s}} \\
& +\frac{\mid \lambda_{2} \mu_{2}(t)\left\|p_{f}\right\| \psi(\|u\|)}{\Gamma\left(\sigma_{2}+\alpha\right)} \int_{1}^{\eta_{2}}\left(\log \frac{\eta_{2}}{s}\right)^{\sigma_{2}+\alpha-1}\left(\frac{(\log s)^{\beta}}{\Gamma(\beta+1)}\right)^{q-1} \frac{\mathrm{ds}}{\mathrm{s}} \\
& +\frac{\left|a_{2} \mu_{2}(t)\right|\left\|p_{f}\right\| \psi(\|u\|)}{\Gamma(\alpha)} \int_{1}^{T}\left(\log \frac{T}{s}\right)^{\alpha-1}\left(\frac{(\log s)^{\beta}}{\Gamma(\beta+1)}\right)^{q-1} \frac{\mathrm{ds}}{\mathrm{s}} \\
& +\frac{\left|b_{2} \mu_{2}(t)\right|\left\|p_{f}\right\| \psi(\|u\|)}{\Gamma(\alpha-\gamma)} \int_{1}^{T}\left(\log \frac{T}{s}\right)^{\alpha-\gamma-1}\left(\frac{(\log s)^{\beta}}{\Gamma(\beta+1)}\right)^{q-1} \frac{\mathrm{ds}}{\mathrm{s}} \\
& =\frac{\left\|p_{f}\right\| \psi(\|u\|)}{\Gamma(\beta+1)^{q-1} \Gamma(\alpha)} \int_{1}^{t}\left(\log \frac{t}{s}\right)^{\alpha-1}(\log s)^{\beta(q-1)} \frac{\mathrm{ds}}{\mathrm{s}} \\
& +\frac{\mid \mu_{1}(t)\left\|p_{f}\right\| \psi(\|u\|)}{\Gamma(\beta+1)^{q-1} \Gamma\left(\sigma_{1}+\alpha\right)} \int_{1}^{\eta_{1}}\left(\log \frac{\eta_{1}}{s}\right)^{\sigma_{1}+\alpha-1}(\log s)^{\beta(q-1)} \frac{\mathrm{ds}}{\mathrm{s}} \\
& +\frac{\left|\lambda_{2} \mu_{2}(t)\left\||| p_{f}\right\| \psi(\|u\|)\right.}{\Gamma(\beta+1)^{q-1} \Gamma\left(\sigma_{2}+\alpha\right)} \int_{1}^{\eta_{2}}\left(\log \frac{\eta_{2}}{s}\right)^{\sigma_{2}+\alpha-1}(\log s)^{\beta(q-1)} \frac{\mathrm{ds}}{\mathrm{s}} \\
& +\frac{\left|a_{2} \mu_{2}(t)\right|\left\|p_{f}\right\| \psi(\|u\|)}{\Gamma(\beta+1)^{q-1} \Gamma(\alpha)} \int_{1}^{T}\left(\log \frac{T}{s}\right)^{\alpha-1}(\log s)^{\beta(q-1)} \frac{\mathrm{ds}}{\mathrm{s}} \\
& +\frac{\left|b_{2} \mu_{2}(t)\right|\left\|p_{f}\right\| \psi(\|u\|)}{\Gamma(\beta+1)^{q-1} \Gamma(\alpha-\gamma)} \int_{1}^{T}\left(\log \frac{T}{s}\right)^{\alpha-\gamma-1}(\log s)^{\beta(q-1)} \frac{\mathrm{ds}}{\mathrm{s}} \\
& =\frac{\left\|p_{f}\right\| \psi(\|u\|) \Gamma(\beta(q-1)+1)}{\Gamma(\beta+1)^{q-1}}\left\{\frac{(\log T)^{\alpha+\beta(q-1)}\left(1+\left|a_{2}\right| \tilde{\mu_{2}}\right)}{\Gamma(\alpha+\beta(q-1)+1)}\right. \\
& +\frac{\tilde{\mu_{1}}\left(\log \eta_{1}\right)^{\sigma_{1}+\alpha+\beta(q-1)}}{\Gamma\left(\sigma_{1}+\alpha+\beta(q-1)+1\right)}+\tilde{\mu_{2}}\left[\frac{\left|\lambda_{2}\right|\left(\log \eta_{2}\right)^{\sigma_{2}+\alpha+\beta(q-1)}}{\Gamma\left(\sigma_{2}+\alpha+\beta(q-1)+1\right)}\right. \\
& \left.\left.+\frac{\left|b_{2}\right|(\log T)^{\alpha-\gamma+\beta(q-1)}}{\Gamma(\alpha-\gamma+\beta(q-1)+1)}\right]\right\} \\
& \leq \mathcal{M}_{f} \psi(R) \leq R .
\end{aligned}
$$

Thus

$$
\|\mathcal{N} u\| \leq R .
$$

This proves that $\mathcal{N}$ transforms the ball $B_{R}$ into itself. Furthermore for any $u \in B_{R}$ and $t \in J$, we have

$$
\begin{gathered}
\left\|(\mathcal{N} u)^{\prime}(t)\right\| \\
\leq \frac{1}{\Gamma(\alpha-1)} \int_{1}^{t}\left(\log \frac{t}{s}\right)^{\alpha-2} \phi_{q}\left(\int_{1}^{s} \frac{\left(\log \frac{s}{\tau}\right)^{\beta-1}}{\Gamma(\beta)} \phi_{p}\left(p_{f}(\tau) \psi(\|u(\tau)\|)\right) \frac{\mathrm{d} \tau}{\tau}\right) \frac{\mathrm{d} s}{\mathrm{~s}} \\
+\frac{\left|\lambda_{1} \Delta_{2}\right|}{T \Gamma\left(\sigma_{1}+\alpha\right)} \int_{1}^{\eta_{1}}\left(\log \frac{\eta_{1}}{s}\right)^{\sigma_{1}+\alpha-1} \phi_{q}\left(\int_{1}^{s} \frac{\left(\log \frac{s}{\tau}\right)^{\beta-1}}{T \Gamma(\beta)} \phi_{p}\left(p_{f}(\tau) \psi(\|u(\tau)\|)\right) \frac{\mathrm{d} \tau}{\tau}\right) \frac{\mathrm{d} s}{\mathrm{~s}}
\end{gathered}
$$




$$
\begin{aligned}
& +\frac{\left|\lambda_{2} \Delta_{4}\right|}{T \Gamma\left(\sigma_{2}+\alpha\right)} \int_{1}^{\eta_{2}}\left(\log \frac{\eta_{2}}{s}\right)^{\sigma_{2}+\alpha-1} \phi_{q}\left(\int_{1}^{s} \frac{\left(\log \frac{s}{\tau}\right)^{\beta-1}}{\Gamma(\beta)} \phi_{p}\left(p_{f}(\tau) \psi(\|u(\tau)\|)\right) \frac{\mathrm{d} \tau}{\tau}\right) \frac{\mathrm{d} s}{\mathrm{~s}} \\
& +\frac{\left|a_{2} \Delta_{4}\right|}{T \Gamma(\alpha)} \int_{1}^{T}\left(\log \frac{T}{s}\right)^{\alpha-1} \phi_{q}\left(\int_{1}^{s} \frac{\left(\log \frac{s}{\tau}\right)^{\beta-1}}{\Gamma(\beta)} \phi_{p}\left(p_{f}(\tau) \psi(\|u(\tau)\|)\right) \frac{\mathrm{d} \tau}{\tau}\right) \frac{\mathrm{d} \mathrm{s}}{\mathrm{s}} \\
& +\frac{\left|b_{2} \Delta_{4}\right|}{T \Gamma(\alpha-\gamma)} \int_{1}^{T}\left(\log \frac{T}{s}\right)^{\alpha-\gamma-1} \phi_{q}\left(\int_{1}^{s} \frac{\left(\log \frac{s}{\tau}\right)^{\beta-1}}{\Gamma(\beta)} \phi_{p}\left(p_{f}(\tau) \psi(\|u(\tau)\|)\right) \frac{\mathrm{d} \tau}{\tau}\right) \frac{\mathrm{ds}}{\mathrm{s}}
\end{aligned}
$$

Some computations give

$$
\begin{aligned}
\left\|(\mathcal{N} u)^{\prime}(t)\right\| & \leq \frac{\left\|p_{f}\right\| \psi(\|u\|) \Gamma(\beta(q-1)+1)}{\Gamma(\beta+1)^{q-1}}\left\{\frac{(\log T)^{\alpha+\beta(q-1)-1}}{\Gamma(\alpha+\beta(q-1))}\right. \\
& +\frac{\left|\lambda_{1} \Delta_{2}\right|\left(\log \eta_{1}\right)^{\sigma_{1}+\alpha+\beta(q-1)}}{T \Gamma\left(\sigma_{1}+\alpha+\beta(q-1)+1\right)}+\frac{\left|\Delta_{4}\right|}{T}\left[\frac{\left|\lambda_{2}\right|\left(\log \eta_{2}\right)^{\sigma_{2}+\alpha+\beta(q-1)}}{\Gamma\left(\sigma_{2}+\alpha+\beta(q-1)+1\right)}\right. \\
& \left.\left.+\frac{\left|a_{2}\right|(\log T)^{\alpha+\beta(q-1)}}{\Gamma(\alpha+\beta(q-1)+1)}+\frac{\left|b_{2}\right|(\log T)^{\alpha-\gamma+\beta(q-1)}}{\Gamma(\alpha-\gamma+\beta(q-1)+1)}\right]\right\} \\
& =\mathcal{L}_{f} \psi(\|u\|),
\end{aligned}
$$

where $\mathcal{L}_{f}$ is given by (3.10). We shall show that the operator $\mathcal{N}: B_{R} \longrightarrow B_{R}$ satisfies all the assumptions of Theorem 2.2.

For clarity, we will divide the remain of the proof into several steps.

Step 1: $\mathcal{N}: B_{R} \longrightarrow B_{R}$ is continuous. Let $\left\{u_{n}\right\}_{n \in \mathbb{N}}$ be a sequence such that $u_{n} \rightarrow u$ in $B_{R}$. Then, for each $t \in J$,we have

$$
\begin{gathered}
\left\|\left(\mathcal{N} u_{n}\right)(t)-(\mathcal{N} u)(t)\right\| \\
\leq \frac{1}{\Gamma(\alpha)} \int_{1}^{t}\left(\log \frac{t}{s}\right)^{\alpha-1} \phi_{q}\left(\int_{1}^{s} \frac{\left(\log \frac{s}{\tau}\right)^{\beta-1}}{\Gamma(\beta)} \| f\left(\tau, u_{n}(\tau)-f(\tau, u(\tau)) \| \frac{\mathrm{d} \tau}{\tau}\right) \frac{\mathrm{d} s}{\mathrm{~s}}\right. \\
+\frac{\left|\mu_{1}(t)\right|}{\Gamma\left(\sigma_{1}+\alpha\right)} \int_{1}^{\eta_{1}}\left(\log \frac{\eta_{1}}{s}\right)^{\sigma_{1}+\alpha-1} \phi_{q}\left(\int_{1}^{s} \frac{\left(\log \frac{s}{\tau}\right)^{\beta-1}}{\Gamma(\beta)} \| f\left(\tau, u_{n}(\tau)-f(\tau, u(\tau)) \| \frac{\mathrm{d} \tau}{\tau}\right) \frac{\mathrm{d} s}{\mathrm{~s}}\right. \\
+\frac{\left|\lambda_{2} \mu_{2}(t)\right|}{\Gamma\left(\sigma_{2}+\alpha\right)} \int_{1}^{\eta_{2}}\left(\log \frac{\eta_{2}}{s}\right)^{\sigma_{2}+\alpha-1} \phi_{q}\left(\int_{1}^{s} \frac{\left(\log \frac{s}{\tau}\right)^{\beta-1}}{\Gamma(\beta)} \| f\left(\tau, u_{n}(\tau)-f(\tau, u(\tau)) \| \frac{\mathrm{d} \tau}{\tau}\right) \frac{\mathrm{d} s}{\mathrm{~s}}\right. \\
+\frac{\left|a_{2} \mu_{2}(t)\right|}{\Gamma(\alpha)} \int_{1}^{T}\left(\log \frac{T}{s}\right)^{\alpha-1} \phi_{q}\left(\int_{1}^{s} \frac{\left(\log \frac{s}{\tau}\right)^{\beta-1}}{\Gamma(\beta)} \| f\left(\tau, u_{n}(\tau)-f(\tau, u(\tau)) \| \frac{\mathrm{d} \tau}{\tau}\right) \frac{\mathrm{d} s}{\mathrm{~s}}\right. \\
+\frac{\left|b_{2} \mu_{2}(t)\right|}{\Gamma(\alpha-\gamma)} \int_{1}^{T}\left(\log \frac{T}{s}\right)^{\alpha-\gamma-1} \phi_{q}\left(\int_{1}^{s} \frac{\left(\log \frac{s}{\tau}\right)^{\beta-1}}{\Gamma(\beta)} \| f\left(\tau, u_{n}(\tau)-f(\tau, u(\tau)) \| \frac{\mathrm{d} \tau}{\tau}\right) \frac{\mathrm{d} s}{\mathrm{~s}}\right.
\end{gathered}
$$


Since $u_{n} \rightarrow u$ as $n \rightarrow \infty$ and $f, \phi_{q}(\cdot)$ are continuous, then by the Lebesgue dominated convergence theorem, equation (3.12) implies

$$
\left\|\mathcal{N} u_{n}-\mathcal{N} u\right\| \rightarrow 0, \quad \text { as } \quad n \rightarrow \infty .
$$

Step 2: $\mathcal{N}\left(B_{R}\right)$ is bounded and equicontinuous. Since $\mathcal{N}\left(B_{R}\right) \subset B_{R}$ and $B_{R}$ is bounded, then $\mathcal{N}\left(B_{R}\right)$ is bounded. Next, let $t_{1}, t_{2} \in J, t_{1}<t_{2}, u \in B_{R}$. Thus, we have

$$
\left\|\mathcal{N}(u)\left(t_{2}\right)-\mathcal{N}(u)\left(t_{1}\right)\right\| \leq \int_{t_{1}}^{t_{2}}\left\|(\mathcal{N} u)^{\prime}(t)\right\| \mathrm{d} t \leq \mathcal{L}_{f} \psi(R)\left|t_{2}-t_{1}\right|
$$

where $\mathcal{L}_{f}$ is given by (3.10). As $t_{2} \rightarrow t_{1}$, the right-hand side of the above inequality tends to zero.

Step 3:The implication (2.1) holds. Now let $V$ be a subset of $B_{R}$ such that $V \subset$ $\overline{\mathcal{N}(V)} \cup\{0\} . \quad V$ is bounded and equicontinuous and therefore the function $t \rightarrow$ $v(t)=\kappa(V(t))$ is continuous on $J$. By assumption (H3), and the properties of the measure $\kappa$ we have for each $t \in J$.

$$
\begin{aligned}
& v(t) \leq \kappa(\overline{\mathcal{N}(V)} \cup\{0\}) \leq \kappa(\mathcal{N}(V)(t)) \\
& \leq \kappa\left(\frac{1}{\Gamma(\alpha)} \int_{1}^{t}\left(\log \frac{t}{s}\right)^{\alpha-1} \phi_{q}\left(\int_{1}^{s} \frac{\left(\log \frac{s}{\tau}\right)^{\beta-1}}{\Gamma(\beta)} f(\tau, u(\tau)) \frac{\mathrm{d} \tau}{\tau}\right) \frac{\mathrm{d} s}{\mathrm{~s}}\right) \\
& +\kappa\left(\frac{\mu_{1}(t)}{\Gamma\left(\sigma_{1}+\alpha\right)} \int_{1}^{\eta_{1}}\left(\log \frac{\eta_{1}}{s}\right)^{\sigma_{1}+\alpha-1} \phi_{q}\left(\int_{1}^{s} \frac{\left(\log \frac{s}{\tau}\right)^{\beta-1}}{\Gamma(\beta)} f(\tau, u(\tau)) \frac{\mathrm{d} \tau}{\tau}\right) \frac{\mathrm{d} \mathrm{s}}{\mathrm{s}}\right) \\
& +\kappa\left(\frac{\lambda_{2} \mu_{2}(t)}{\Gamma\left(\sigma_{2}+\alpha\right)} \int_{1}^{\eta_{2}}\left(\log \frac{\eta_{2}}{s}\right)^{\sigma_{2}+\alpha-1} \phi_{q}\left(\int_{1}^{s} \frac{\left(\log \frac{s}{\tau}\right)^{\beta-1}}{\Gamma(\beta)} f(\tau, u(\tau)) \frac{\mathrm{d} \tau}{\tau}\right) \frac{\mathrm{ds}}{\mathrm{s}}\right) \\
& +\kappa\left(\frac{a_{2} \mu_{2}(t)}{\Gamma(\alpha)} \int_{1}^{T}\left(\log \frac{T}{s}\right)^{\alpha-1} \phi_{q}\left(\int_{1}^{s} \frac{\left(\log \frac{s}{\tau}\right)^{\beta-1}}{\Gamma(\beta)} f(\tau, u(\tau)) \frac{\mathrm{d} \tau}{\tau}\right) \frac{\mathrm{ds}}{\mathrm{s}}\right) \\
& +\kappa\left(\frac{b_{2} \mu_{2}(t)}{\Gamma(\alpha-\gamma)} \int_{1}^{T}\left(\log \frac{T}{s}\right)^{\alpha-\gamma-1} \phi_{q}\left(\int_{1}^{s} \frac{\left(\log \frac{s}{\tau}\right)^{\beta-1}}{\Gamma(\beta)} f(\tau, u(\tau)) \frac{\mathrm{d} \tau}{\tau}\right) \frac{\mathrm{ds}}{\mathrm{s}}\right) \\
& \leq \frac{1}{\Gamma(\alpha)} \int_{1}^{t}\left(\log \frac{t}{s}\right)^{\alpha-1} \phi_{q}\left(\int_{1}^{s} \frac{\left(\log \frac{s}{\tau}\right)^{\beta-1}}{\Gamma(\beta)} \kappa(f(\tau, V(\tau))) \frac{\mathrm{d} \tau}{\tau}\right) \frac{\mathrm{ds}}{\mathrm{s}} \\
& +\frac{\left|\tilde{\mu}_{1}\right|}{\Gamma\left(\sigma_{1}+\alpha\right)} \int_{1}^{\eta_{1}}\left(\log \frac{\eta_{1}}{s}\right)^{\sigma_{1}+\alpha-1} \phi_{q}\left(\int_{1}^{s} \frac{\left(\log \frac{s}{\tau}\right)^{\beta-1}}{\Gamma(\beta)} \kappa(f(\tau, V(\tau))) \frac{\mathrm{d} \tau}{\tau}\right) \frac{\mathrm{d} s}{\mathrm{~s}} \\
& +\frac{\left|\lambda_{2} \tilde{\mu}_{2}\right|}{\Gamma\left(\sigma_{2}+\alpha\right)} \int_{1}^{\eta_{2}}\left(\log \frac{\eta_{2}}{s}\right)^{\sigma_{2}+\alpha-1} \phi_{q}\left(\int_{1}^{s} \frac{\left(\log \frac{s}{\tau}\right)^{\beta-1}}{\Gamma(\beta)} \kappa(f(\tau, V(\tau))) \frac{\mathrm{d} \tau}{\tau}\right) \frac{\mathrm{ds}}{\mathrm{s}} \\
& +\frac{\left|a_{2} \tilde{\mu}_{2}\right|}{\Gamma(\alpha)} \int_{1}^{T}\left(\log \frac{T}{s}\right)^{\alpha-1} \phi_{q}\left(\int_{1}^{s} \frac{\left(\log \frac{s}{\tau}\right)^{\beta-1}}{\Gamma(\beta)} \kappa(f(\tau, V(\tau))) \frac{\mathrm{d} \tau}{\tau}\right) \frac{\mathrm{ds}}{\mathrm{s}}
\end{aligned}
$$




$$
\begin{aligned}
& +\frac{\left|b_{2} \tilde{\mu}_{2}\right|}{\Gamma(\alpha-\gamma)} \int_{1}^{T}\left(\log \frac{T}{s}\right)^{\alpha-\gamma-1} \phi_{q}\left(\int_{1}^{s} \frac{\left(\log \frac{s}{\tau}\right)^{\beta-1}}{\Gamma(\beta)} \kappa(f(\tau, V(\tau))) \frac{d \tau}{\tau}\right) \frac{\mathrm{ds}}{\mathrm{s}} \\
& \leq \frac{1}{\Gamma(\alpha)} \int_{1}^{t}\left(\log \frac{t}{s}\right)^{\alpha-1} \phi_{q}\left(\int_{1}^{s} \frac{\left(\log \frac{s}{\tau}\right)^{\beta-1}}{\Gamma(\beta)} \phi_{p}\left(p_{f}(\tau) v(\tau)\right) \frac{\mathrm{d} \tau}{\tau}\right) \frac{\mathrm{ds}}{\mathrm{s}} \\
& +\frac{\left|\tilde{\mu}_{1}\right|}{\Gamma\left(\sigma_{1}+\alpha\right)} \int_{1}^{\eta_{1}}\left(\log \frac{\eta_{1}}{s}\right)^{\sigma_{1}+\alpha-1} \phi_{q}\left(\int_{1}^{s} \frac{\left(\log \frac{s}{\tau}\right)^{\beta-1}}{\Gamma(\beta)} \phi_{p}\left(p_{f}(\tau) v(\tau)\right) \frac{\mathrm{d} \tau}{\tau}\right) \frac{\mathrm{d} s}{\mathrm{~s}} \\
& +\frac{\left|\lambda_{2} \tilde{\mu}_{2}\right|}{\Gamma\left(\sigma_{2}+\alpha\right)} \int_{1}^{\eta_{2}}\left(\log \frac{\eta_{2}}{s}\right)^{\sigma_{2}+\alpha-1} \phi_{q}\left(\int_{1}^{s} \frac{\left(\log \frac{s}{\tau}\right)^{\beta-1}}{\Gamma(\beta)} \phi_{p}\left(p_{f}(\tau) v(\tau)\right) \frac{\mathrm{d} \tau}{\tau}\right) \frac{\mathrm{d} s}{\mathrm{~s}} \\
& +\frac{\left|a_{2} \tilde{\mu}_{2}\right|}{\Gamma(\alpha)} \int_{1}^{T}\left(\log \frac{T}{s}\right)^{\alpha-1} \phi_{q}\left(\int_{1}^{s} \frac{\left(\log \frac{s}{\tau}\right)^{\beta-1}}{\Gamma(\beta)} \phi_{p}\left(p_{f}(\tau) v(\tau)\right) \frac{\mathrm{d} \tau}{\tau}\right) \frac{\mathrm{ds}}{\mathrm{s}} \\
& +\frac{\left|b_{2} \tilde{\mu}_{2}\right|}{\Gamma(\alpha-\gamma)} \int_{1}^{T}\left(\log \frac{T}{s}\right)^{\alpha-\gamma-1} \phi_{q}\left(\int_{1}^{s} \frac{\left(\log \frac{s}{\tau}\right)^{\beta-1}}{\Gamma(\beta)} \phi_{p}\left(p_{f}(\tau) v(\tau)\right) \frac{d \tau}{\tau}\right) \frac{\mathrm{ds}}{\mathrm{s}} \\
& \leq \mathcal{M}_{f}\|v\|_{\infty},
\end{aligned}
$$

where $\mathcal{M}_{f}$ is given by (3.9). Which gives

$$
\|v\|_{\infty} \leq \mathcal{M}_{f}\|v\|_{\infty}
$$

This means that

$$
\|v\|_{\infty}\left(1-\mathcal{M}_{f}\right) \leq 0 .
$$

From (3.11), we get $\|v\|_{\infty}=0$, that is, $v(t)=\kappa(V(t))=0$, for each $t \in J$, and then $V(t)$ is relatively compact in $E$. In view of the Ascoli-Arzela theorem, $V$ is relatively compact in $B_{R}$. Applying Theorem 2.2 we conclude that $\mathcal{N}$ has a fixed point which is a solution of the problem (1.1)-(1.2).

\section{An Example}

In this section, we give an example to illustrate the usefulness of our main result. Let

$$
E=c_{0}=\left\{u=\left(u_{1}, u_{2}, \ldots, u_{n}, \ldots\right): u_{n} \rightarrow 0(n \rightarrow \infty)\right\},
$$

be the Banach space of real sequences converging to zero, endowed its usual norm

$$
\|u\|_{\infty}=\sup _{n \geq 1}\left|u_{n}\right| .
$$

Example 4.1. Consider the following fractional problem posed in $c_{0}$ :

$$
{ }_{H}^{C} \mathcal{D}_{1}^{\frac{1}{2}}\left(\phi_{p}\left[{ }_{H}^{C} \mathcal{D}_{1}^{\frac{7}{4}} u(t)\right]\right)=f(t, u(t)), t \in J:=[1, e],
$$


supplemented with boundary conditions

$$
\begin{aligned}
u(1)+{ }_{H}^{C} \mathcal{D}_{1}^{\frac{1}{2}} u(1) & ={ }^{H}{ }^{\frac{5}{2}} u\left(\frac{5}{4}\right), 0<\gamma \leq 1 \\
u(e)+{ }_{H}^{C} \mathcal{D}_{1}^{\frac{1}{2}} u(e) & ={ }^{H_{\mathcal{I}}} \frac{7}{2} u\left(\frac{3}{2}\right), 1<\eta_{1}, \eta_{2}<T \\
{ }_{H}^{C} \mathcal{D}_{1}^{\frac{7}{4}} u(1) & =(0,0, \ldots, 0, \ldots) .
\end{aligned}
$$

Note that, this problem is a particular case of BVP (1.1)-(1.2), where

$$
\begin{aligned}
\alpha & =\frac{7}{4}, \beta=\gamma=\frac{1}{2}, T=e \\
a_{1} & =b_{1}=a_{2}=b_{2}=\lambda_{1}=\lambda_{2}=1 \\
\sigma_{1} & =\frac{5}{2}, \sigma_{2}=\frac{7}{2}, \eta_{1}=\frac{5}{4}, \eta_{2}=\frac{3}{2} \\
p & =2, q=2
\end{aligned}
$$

and $f: J \times c_{0} \longrightarrow c_{0}$ given by

$$
f(t, u)=\left\{\phi_{p}\left(\frac{1}{\left(t^{2}+2\right)^{2}}\left(\frac{1}{n^{2}}+\sin \left|u_{n}\right|\right)\right)\right\}_{n \geq 1}, \quad \text { for } t \in J, u=\left\{u_{n}\right\}_{n \geq 1} \in c_{0} .
$$

It is clear that condition (H1) holds, and as

$$
\begin{aligned}
\|f(t, u)\| & =\left\|\phi_{p}\left(\frac{1}{\left(t^{2}+2\right)^{2}}\left(\frac{1}{n^{2}}+\sin \left|u_{n}\right|\right)\right)\right\| \\
& \leq \phi_{p}\left(\frac{1}{\left(t^{2}+2\right)^{2}}(1+\|u\|)\right) \\
& =\phi_{p}\left(p_{f}(t) \psi(\|u\|)\right) .
\end{aligned}
$$

Therefore, the assumption (H2) of the Theorem 3.1 is satisfied with $p_{f}(t)=\frac{1}{\left(t^{2}+2\right)^{2}}, t \in J$, and $\psi(u)=1+u, u \in[0, \infty)$. On the other hand, for any bounded set $D \subset c_{0}$, we have

$$
\kappa(f(t, D)) \leq \frac{1}{\left(t^{2}+2\right)^{2}} \kappa(D), \text { for each } t \in J .
$$

Hence (H3) is satisfied. We shall check that condition (3.11) is satisfied. Using the Matlab program, we can find

$$
\mathcal{M}_{f}=0.4228<1
$$

and

thus

$$
(1+R) \mathcal{M}_{f}<R
$$

$$
R>\frac{\mathcal{M}_{f}}{1-\mathcal{M}_{f}}=0.7326,
$$

Then $R$ can be chosen as $R=1 \geq 0.7326$. Consequently, Theorem 3.1 implies that the problem (4.1)-(4.2) has at least one solution $u \in C\left(J, c_{0}\right)$.

\section{Conclusions}

We have proved the existence of solutions for Caputo-Hadamard fractional differential equations with $\mathrm{p}$-Laplacian operator in a given Banach space. The problem is issued by applying Mönch's fixed point theorem combined with the technique of measures noncompactness. We also provide an example to make our results clear. 


\section{R E F E R E N C E S}

1. S. Abbas, M. Benchohra and J. Henderson: Weak Solutions for Implicit Fractional Differential Equations of Hadamard Type. Adv. Dyn. Syst. Appl. 111 (2016) 1-13.

2. S. Abbas, M. Benchohra, N. Hamidi and J Henderson: Caputo-Hadamard fractional differential equations in Banach spaces. Fract. Calc. Appl. Anal. 214 (2018) 1027-1045.

3. Y. Adjabi, F. Jarad, D. Baleanu, T. Abdeljawad: On Cauchy problems with Caputo Hadamard fractional derivatives. J. Comput. Anal. Appl. 214 (2016) 661-681.

4. R. P. Agarwal, M. Benchohra and D. Seba: On the Application of Measure of noncompactness to the existence of solutions for fractional differential equations. Results. Math. $\mathbf{5 5}$ (2009) 221-230.

5. A. Ahmadkhanlu: Existence and uniquensess for a class of fractional differential Equations with an integral fractional boundary condition. Filomat 315 (2017) 1241-1249.

6. J. P. Aubin, I. Ekeland: Applied Nonlinear Analysis. John Wiley \& Sons, New York (1984).

7. A. Ardjouni, A. Djoudi: Positive solutions for nonlinear Caputo-Hadamard fractional differential equations with integral boundary conditions. Open J. Math. Anal. 31 (2019) 62-69.

8. Y. Arioua, N. Benhamidouche: Boundary value problem for Caputo-Hadamard fractional differential equations. Surv. Math. Appl. 12 (2017) 103-115.

9. C. BAI: Existence and uniqueness of solutions for fractional boundary value problems with p-Laplacian operator. Adv. Difference Equ. 2018, Paper No. 4, 12 pp.

10. J. BAnas̀ AND K. GoeBEl: Measures of Noncompactness in Banach Spaces. Marcel Dekker, New York, 1980.

11. J. Banaś, M. Jleli, M. Mursaleen. B. Samet, C. Vetro: Advances in Nonlinear Analysis via the Concept of Measure of Noncompactness. Springer, singpagor 2017.

12. M. Benchohra, J. Henderson And D. Seba: Measure of noncompactness and fractional differential equations in Banach spaces. Commun. Appl. Anal. 124 (2008) 419-427.

13. M. Benchohra, S. Hamani, S.K. Ntouyas: Boundary value problems for differential equations with fractional order and nonlocal conditions. Nonlinear Anal. 71 7-8 (2009) 2391-2396.

14. M. Benchohra AND FAtima-Zohra Mostefai: Weak solutions for nonlinear fractional differential equations with integral boundary conditions in Banach spaces. Opuscula Math. 321 (2012) 31-40.

15. M. Benchohra, S. Bouriah, J.J. Nieto: Existence of periodic solutions for nonlinear implicit Hadamard's fractional differential equations. Rev. R. Acad. Cienc. Exactas Fís. Nat. Ser. A Mat. RACSAM 1121 (2018) 25-35.

16. W. Benhamida, S. Hamani, J. Henderson: Boundary Value Problems For CaputoHadamard Fractional Differential Equations. Adv. Theory Nonlinear Anal. Appl. 2 (2018) 138-145.

17. W. Benhamida, J. R. Graef, S. Hamania: Boundary value problems for Hadamard fractional differential equations with nonlocal multi-point boundary conditions. Fract. Differ. Calc. 81 (2018) 165-176.

18. A. Benlabbes, M. Benbachir, M. Lakrib: Boundary value problems for nonlinear fractional differential equations. Facta Univ. Ser. Math. Inform. 302 (2015) 157-168.

19. Z. Dahmani, M. A. Abdellaoui: New results for a weighted nonlinear system of fractional integro-differential equations. Facta Univ. Ser. Math. Inform. 293 (2014) 233-242.

20. Z. Dahmani, A. TAeB: New existence and uniqueness results for hight dimensional fractional differential systems. Facta Univ. Ser. Math. Inform. 303 (2015) 281-293.

21. Z. Dahmani, A. TAieb, N. Bedjaoui: Solvability and Stability for nonlinear fractional integrodifferential systems of hight fractional orders. Facta Univ. Ser. Math. Inform. 313 (2016) 629-644.

22. X. Dong, Z. Bai And S. Zhang: Positive solutions to boundary value problems of $\mathrm{p}$-Laplacian with fractional derivative. Bound. Value Probl. 2017, Paper No. 5, 15 pp. 
23. F. T. Fen, I. Y. Karacac, O. B. Ozenc: Positive Solutions of Boundary Value Problems for p-Laplacian Fractional Differential Equations. Filomat 315 (2017) 1265-1277

24. Y. Y. Gambo, F. Jarad, D. Baleanu, T. Abdeljawad: On Caputo modification of the Hadamard fractional derivatives. Adv. Difference Equ. 2014, 2014:10, 12 pp.

25. J. R. Graef, N. Guerraiche and S. Hamani, Boundary value problems for fractional differential inclusions with Hadamard type derivatives in Banach spaces. Stud. Univ. Babeş-Bolyai Math. 624 (2017) 427-438.

26. A. Guezane-Lakoud, R. Khaldi: Solvability of a fractional boundary value problem with integral condition. Nonlinear Anal. 754 (2012) 2692-2700.

27. J. HADAmARD: Essai sur l'etude des fonctions donnees par leur developpment de Taylor. J. Mat. Pure Appl. Ser. 8 (1892) 101-186.

28. R. Hilfer: Application of fractional calculus in physics. New Jersey: World Scientific, (2001).

29. M. HouAs AND M. BEZzIOU: Existence and stability resuts for fractional differential equations with two Caputo fractional derivatives. Facta Univ. Ser. Math. Inform. 342 (2019) 341-357.

30. F. Jarad, D. Baleanu AND A. ABdelJawad: Caputo-type modification of the Hadamard fractional derivatives. Adv. Difference Equ. 2012, 2012:142, 8 pp.

31. LS LeIBEnSON: General problem of the movement of a compressible fluid in a porous medium. Izv. Akad. Nauk Kirg.SSR, Ser. Biol. Nauk. 9 (1983) 7-10.

32. N. A. Khan,A. Ara, A. Mahmood: Approximate solution of time-fractional chemical engineering equations: a comparative study. Int. J. Chem. Reactor Eng. 8 (2010) Article A19.

33. A. A. Kilbas, H. M. Srivastava, and J. J. Trujillo: Theory and Applications of Fractional Differential Equations, vol. 204 of North-Holland Mathematics Sudies Elsevier Science B.V. Amsterdam the Netherlands, 2006.

34. X. LIU, M. JIA, X. XIANG: On the solvability of a fractional differential equation model involving the p-Laplacian operator. Comput. Math. Appl. 6410 (2012) 3267-3275.

35. H. LU, Z. HAN, S. SUN Multiplicity of positive solutions for Sturm-Liouville boundary value problems of fractional differential equations with p-Laplacian. Bound. Value Probl. 2014, 2014:26, 17 pp.

36. H. MöNCH: Boundary value problems for nonlinear ordinary differential equations of second order in Banach spaces. Nonlinear Anal. 45 (1980) 985-999.

37. K. B. Oldham: Fractional differential equations in electrochemistry. Adv. Eng. Softw. 411 (2010) 9-12.

38. I. Podlubny: Fractional Differential Equations, Academic Press, San Diego (1993).

39. H. RebAi, D. SebA: Weak Solutions for Nonlinear Fractional Differential Equation with Fractional Separated Boundary Conditions in Banach Spaces. Filomat 323 (2018) 1117-1125.

40. S. SchwabiK Y. Guoju: Topics in Banach Spaces Integration, Series in Real Analysis 10, World Scientific, Singapore, 2005.

41. J. TAN AND M. Li: Solutions of fractional differential equations with $\mathrm{p}$-Laplacian operator in Banach spaces. Bound. Value Probl. 2018, Paper No. 15, 13 pp.

42. V. E. Tarasov, Fractional Dynamics: Application of Fractional Calculus to Dynamics of Particles, Fields and Media. Springer, Heidelberg \& Higher Education Press, Beijing, 2010.

43. J. Tariboon, A. Cuntavepanit, S.K. Ntouyas, W. Nithiarayaphaks: Separated boundary value problems of sequential Caputo and Hadamard fractional differential equations. J. Funct. Spaces 2018, Art. ID 6974046, 8 pp.

44. J. Sabatier, O.P. Agrawal, J.A.T. Machado: Advances in Fractional Calculus-Theoretical Developments and Applications in Physics and Engineering. Dordrecht: Springer, 2007.

45. E. ZEIDLER: Nonlinear functional analysis and its applications. $I I / B$, translated from the German by the author and Leo F. Boron, Springer-Verlag, New York, 1990. 
Choukri Derbazi

Faculty of Science

Laboratory of Mathematics And Applied Sciences University of Ghardaia 47000. Algeria

choukriedp@yahoo.com 